\title{
Avaliação da pesquisa de anticorpos antifosfolipídios para o diagnóstico da síndrome antifosfolípide
}

Paula Gonçalves Perches ${ }^{1}$, Daniela Pezzutti Domingues ${ }^{1}$, Andréia Latanza Gomes ${ }^{2}$, Augusta Morgado Ribeiro ${ }^{2}$, Felipe Moraes Toledo Pereira ${ }^{2}$, Ivan Elias Rassi ${ }^{3}$, Alexandre Wagner Silva de Souza ${ }^{4}$

\begin{abstract}
RESUMO
Objetivo: Determinar a prevalência de anticoagulante lúpico (LAC) e dos isótipos de anticardiolipina (ACL) e suas eventuais associações clínicas. Pacientes e Métodos: Estudo retrospectivo que avaliou manifestações clínicas e laboratoriais em indivíduos que apresentaram positividade para anticorpos antifosfolipídios no Hospital Edmundo Vasconcelos entre março de 2005 e junho de 2006. Resultados: Cento e seis indivíduos (média de idade 42,2 $\pm 14,1$ anos, $84 \%$ do sexo feminino) foram incluídos no estudo. A prevalência de trombose foi de 17,9\% (19/106) e de morbidade gestacional foi de 12,3\% (13/106). O diagnóstico de Síndrome Antifosfolípide (SAF) foi feito em 23,6\% (25/106), sendo primária em $68 \%$ (17/25) e secundária em 32\% (8/25). A prevalência de ACL foi de 97,1\% (103/106) e de LAC foi de 11,4\% (5/44 dos exames realizados). ACL isótipos IgM, IgG e IgA foram encontrados em 100\%, 23,3\% e 4,9\% dos 103 soros ACL positivos, respectivamente. Para o diagnóstico de SAF, a ACL IgM apresentou sensibilidade de $92 \%$ e especificidade de $1,2 \%$, enquanto a ACL IgG teve sensibilidade de $40 \%$ e especificidade de $82,5 \%$. A ausência de ACL IgG teve alto valor preditivo negativo $(81,4 \%$ ) para SAF. O LAC apresentou sensibilidade de $18,7 \%$ e especificidade de $92,8 \%$. A curva Receiver Operating Characteristic (ROC) demonstrou maior área abaixo da curva para ACL IgG e LAC. Conclusão: Em amostra aleatória de indivíduos com anticorpos antifosfolipídios positivos, ACL IgG e LAC apresentaram maior especificidade para o diagnóstico de SAF, que se caracterizou pela maior prevalência de trombose.
\end{abstract}

Palavras-chave: síndrome antifosfolípide, anticorpos anticardiolipina, anticoagulante lúpico, trombose, perda gestacional.

INTRODUÇÃO

A síndrome antifosfolípide (SAF) caracteriza-se pela presença de anticorpos antifosfolipídios associada à trombose arterial, venosa e/ou microangiopática, morbidade gestacional recorrente e/ou trombocitopenia. Pode ser primária ou secundária a outras doenças autoimunes, principalmente ao lúpus eritematoso sistêmico (LES), a doenças infecciosas crônicas ou a neoplasias. ${ }^{1,2}$

Anticorpos antifosfolipídios são autoanticorpos que têm como alvos fosfolipídios de membrana celular e/ou proteínas combinadas a fosfolipídios, como a beta-2 glicoproteína I $\left(\beta_{2}\right.$-GPI) e a protrombina. Os principais anticorpos antifosfolipídios são o anticoagulante lúpico (LAC) e a anticardiolipina (ACL), que pode ser das classes $\operatorname{IgG}, \operatorname{IgM}$ ou $\operatorname{IgA} .{ }^{2}$ A presença de LAC ou de altos títulos de ACL são importantes fatores de risco independentes para tromboses. ${ }^{3}$ Além disso, esses autoanticorpos também auxiliam no diagnóstico de SAF, compondo os critérios de classificação dessa entidade., ${ }^{4,5}$

Neste estudo, procuramos avaliar a prevalência de manifestações clínicas associadas à presença de anticorpos anti-

Recebido em 07/08/2008. Aprovado, após revisão, em 30/01/2009. Declaramos a inexistência de conflitos de interesse, exceto para o autor Alexandre Wagner Silva Souza, que informou os seguintes conflitos de interesse: Recebeu honorários para ministrar a aula "O Sistema imunológico" e financiamento para participar do ACR 2008 - Roche Brasil.

1. Serviço de Clínica Médica do Hospital Professor Edmundo Vasconcellos. 2. Disciplina de Reumatologia da UNIFESP

1. Médica-Residente de clínica médica do Hospital Professor Edmundo Vasconcellos

2. Graduandas(o) em medicina da UNIFESP

3. Chefe do Laboratório do Hospital Professor Edmundo Vasconcellos

4. Assistente Doutor da Disciplina de Reumatologia da UNIFESP

Endereço para correspondência: Alexandre Wagner Silva de Souza. Rua Botucatu, 740. Disciplina de Reumatologia. $3^{\circ}$ andar. Vila Clementino. São Paulo-SP. CEP: 04023-900. E-mail: alexandre_wagner@uol.com.br 
fosfolipídios, comparar a prevalência dessas manifestações e de diferentes anticorpos antifosfolipídios, em pacientes com e sem SAF, e verificar o desempenho de diferentes testes para o diagnóstico da síndrome.

\section{PACIENTES E MÉTODOS}

Foi realizado estudo retrospectivo por meio de busca ativa de resultados de exames positivos para ACL e LAC no Laboratório de Análises Clínicas do Hospital Professor Edmundo Vasconcelos no período de março de 2005 a junho de 2006. Posteriormente foram analisados os prontuários desses indivíduos. Foram incluídos 106 pacientes de acordo com o seguinte critério: presença de ACL superior a $10 \mathrm{MPL}$, APL e/ou GPL e/ou positividade do LAC. Os pacientes com anticorpos antifosfolipídios foram divididos em dois grupos: aqueles que preencheram os Critérios de Classificação de Sapporo para $\mathrm{SAF}^{4}$ e aqueles que não preencheram esses critérios. Os grupos foram comparados em relação às manifestações clínicas, e a contribuição de cada anticorpo antifosfolipídio para o diagnóstico de SAF foi avaliada estatisticamente.

A pesquisa de ACL foi realizada por ensaio imunoenzimático (ELISA) com o soro dos participantes, utilizando kits da INOVA Diagnostics. Os resultados foram emitidos em unidades MPL, APL e GPL para IgM, IgA e IgG, respectivamente. O teste de LAC foi realizado com o plasma dos indivíduos com os seguintes testes: tempo de tromboplastina parcial ativada (TTPa) e tempo de coagulação com o veneno da cobra Russel diluído (dRVVT), de acordo com diretrizes internacionais. ${ }^{6}$

O estudo foi aprovado pela Comissão de Ética do Hospital Professor Edmundo Vasconcelos.

\section{ANÁLISE ESTATÍSTICA}

Os testes de qui quadrado ou de Fisher foram empregados para analisar variáveis categóricas, enquanto os testes $t$ de Student ou de Mann-Whitney foram utilizados para comparar variáveis numéricas. Os coeficientes de kurtosis e skewness foram calculados para verificar se as variáveis numéricas apresentavam distribuição normal. Foram considerados significativos os valores de $\mathrm{P}$ inferiores a 0,05 .

A correlação dos níveis de ACL IgM, IgA e IgG entre si e com a presença de LAC foi realizada pelo coeficiente de correlação de Spearmann. Para cada teste diagnóstico foram calculados sensibilidade, especificidade, valor preditivo positivo (VPP) e valor preditivo negativo (VPN), além da construção da curva ROC (Receiver Operating Characteristic) com a área abaixo da curva e intervalo de confiança de 95\% (IC 95\%).
O padrão-ouro utilizado para o diagnóstico de SAF foram os Critérios de Classificação de Sapporo.

O risco de trombose associado a cada teste diagnóstico do estudo foi feito por regressão logística, stepwise. Os resultados foram expressos em razão de chances com intervalo de confiança (IC) de 95\%.

\section{RESULTADOS}

Foram encontrados testes positivos para anticorpos antifosfolipídios em 106 indivíduos. Os participantes do estudo tinham média de idade de 42,2 anos com desvio-padrão (DP) de 14,1 anos, na faixa entre 10 e 80 anos, e a maioria era do sexo feminino (84\%).

Episódios de trombose foram diagnosticados em 17,9\% (19 dos 106 pacientes), sendo trombose venosa presente em 73,7\% $(14 / 19)$ e arterial em 31,6\% (6/19). A morbidade gestacional foi observada em 12,3\% (13/106 pacientes). Os critérios de Classificação de Sapporo para SAF foram preenchidos em $23,6 \%$ dos casos (25/106 pacientes), dentre os quais $68 \%$ apresentaram a forma primária e $32 \%$ a forma secundária da doença, seis deles com o diagnóstico de LES, um com hepatite C crônica e um com infecção crônica pelo HIV.

A prevalência de ACL foi de 97,1\% (103/106 pacientes) e a de LAC foi de 11,4\% (5 dos 44 pacientes avaliados). ACL IgM, IgG e IgA foram encontradas, respectivamente, em 100\%, $23,3 \%$ e $4,9 \%$ dos soros ACL positivos (Tabela 1). ACL IgM, IgG e IgA tiveram títulos médios de 35,5 MPL, 32,9 GPL e 19,1 APL, respectivamente.

Pacientes com e sem SAF apresentaram dados semelhantes em relação à idade $(45,2 \pm 13,1$ versus $41,2 \pm 14,3$ anos, $\mathrm{P}=$ $0,198)$, ao sexo feminino ( $84 \%$ versus $83,9 \%, \mathrm{P}=0,995)$ e à frequência de ACL IgM ( $92 \%$ versus $98,7 \%, \mathrm{P}=0,075)$ e ACL IgA ( $4 \%$ versus $4,9 \%, \mathrm{P}=0,889)$. No entanto, a prevalência ( $40 \%$ versus $17,2 \%, \mathrm{P}=0,019$ ) e os níveis médios de ACL IgG $(38,8 \pm 21,8$ GPL versus $28,7 \pm 28,7$ GPL, $\mathrm{P}=0,038)$ foram maiores em pacientes com $\mathrm{SAF}$ em relação àqueles sem $\mathrm{SAF}$. Foi observado também um número significativamente maior de indivíduos com níveis médios de ACL IgG acima de 40 GPL entre os pacientes com $\mathrm{SAF}$ em relação àqueles sem $\mathrm{SAF}(16 \%$ versus $2,5 \%, \mathrm{P}=0,001)$. Quanto à $\mathrm{ACL}$ da classe IgM, não houve diferença significativa entre os grupos com e sem SAF em relação ao número de indivíduos com níveis médios superiores a 40MPL (24\% versus $13,6 \%, \mathrm{P}=0,215)$ (Tabela 1$)$.

Dos 44 pacientes submetidos à pesquisa de LAC, cinco apresentaram o teste positivo $(11,3 \%)$, sendo três com diagnóstico de SAF, segundo os Critérios de Classificação de Sapporo, $\mathrm{P}=0,336$. Pacientes com SAF apresentaram maior frequência 
Tabela 1

Dados demográficos, laboratoriais e clínicos dos pacientes antifosfolípide positivos com e sem SAF

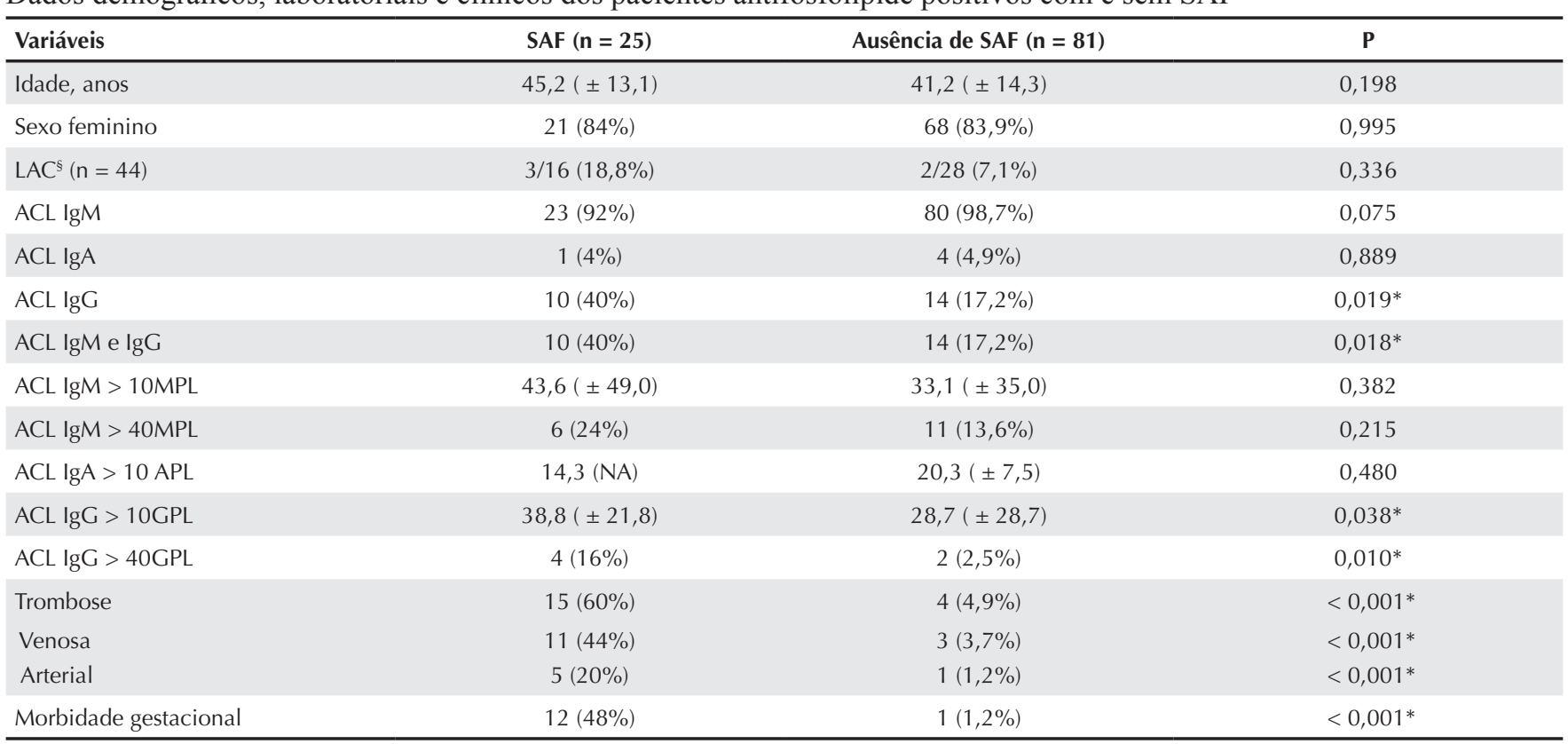

$\mathrm{NA}=$ Não aplicável; $\mathrm{ACL}=$ anticardiolipina; $\mathrm{LAC}=$ anticoagulante lúpico. ${ }^{\circledR} 0$ teste de $\mathrm{LAC}$ foi realizado em 44 dos pacientes inclú́dos no estudo. ${ }^{*}$ Diferença estatisticamente significativa.

de ACL IgM e IgG concomitantes (40\% versus $17,2 \%$, P $=0,018)$ em relação àqueles sem SAF. Pacientes com SAF apresentaram maior prevalência de trombose arterial $(20 \%$ versus $1,2 \%, \mathrm{P}<0,001)$ trombose venosa $(44 \%$ versus $3,7 \%$, $\mathrm{P}<0,001)$ e de morbidade gestacional ( $48 \%$ versus $1,2 \%$, $\mathrm{P}$ $<0,001)$ em comparação com indivíduos que apresentaram anticorpos antifosfolipídios sem preencherem critérios de Classificação para SAF (Tabela 1).

Em análise multivariada, os anticorpos antifosfolipídios LAC e ACL (IgM, IgA e IgG) não se mostraram associados de forma significativa a um maior risco de trombose. A ACL $\mathrm{IgG}$ apresentou risco de 2,4 vezes de trombose, mas o IC $95 \%$ de 0,4 a 13,7 não foi significativo $(P=0,315)$.

A ACL IgM apresentou sensibilidade de $92 \%$ e especificidade de $1,2 \%$ para o diagnóstico de SAF. O VPP foi de $22,3 \%$ e o VPN foi de $33,3 \%$ para ACL IgM. A curva ROC demonstrou área abaixo da curva de 0,466 com IC $95 \%$ de 0,332-0,600 (Figura 1). A ACL IgG apresentou sensibilidade de $40 \%$ e especificidade $\backslash$ de $82,5 \%$ para diagnóstico de SAF. O VPP para ACL IgG foi de $41,6 \%$ e o VPN de $81,4 \%$. A curva ROC demonstrou área abaixo da curva de $0,613 \mathrm{com}$ IC 95\% de 0,479-0,746 (Figura 2). O LAC apresentou sensibilidade de $12 \%$ e especificidade de $97,5 \%$ para o diagnóstico de SAF. O VPP foi de $60 \%$ e o VPN foi de $78,2 \%$. A curva ROC demonstrou área abaixo da curva de 0,558 com IC 95\% de 0,376-0,740 (Figura 3). A prevalência muito baixa de ACL IgA não permitiu análise similar quanto ao desempenho deste teste diagnóstico.

\section{DISCUSSÃO}

Neste estudo, foram levantados todos os exames de ACL IgM, IgG e IgA, além de LAC, realizados em laboratório de hospital geral num período de 16 meses. Os resultados da pesquisa de anticorpos antifosfolipídios obtidos foram confrontados com as manifestações clínicas e antecedentes pessoais apresentados pelos participantes do estudo e com os critérios de classificação de Sapporo para SAF. ${ }^{4}$ ACL da classe IgG e LAC foram os testes que se mostraram mais específicos para o diagnóstico de SAF, enquanto a ACL da classe IgM se mostrou muito sensível e pouco específica. Pacientes com o diagnóstico de SAF apresentaram maiores títulos de ACL da classe IgG. Entretanto, o desenho retrospectivo do presente estudo impede que se emitam conclusões sobre causalidade.

A padronização de testes diagnósticos para a pesquisa de anticorpos antifosfolipídios tem sido um desafio, pois diversos estudos demonstraram grande variedade de resultados obtidos entre diferentes kits comerciais e técnicas in-house de ELISA entre laboratórios para a pesquisa de ACL. ${ }^{7-10}$ Nesse sentido, seria interessante estender nossos achados utilizando kits co- 


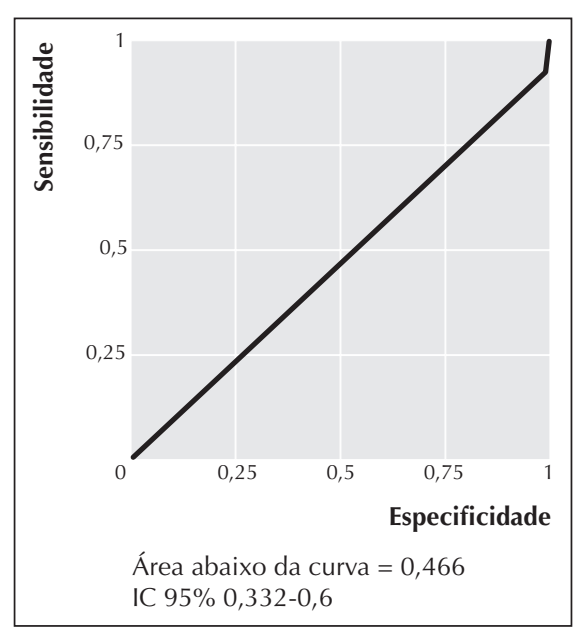

Figura 1. Curva ROC para ACL IgM.

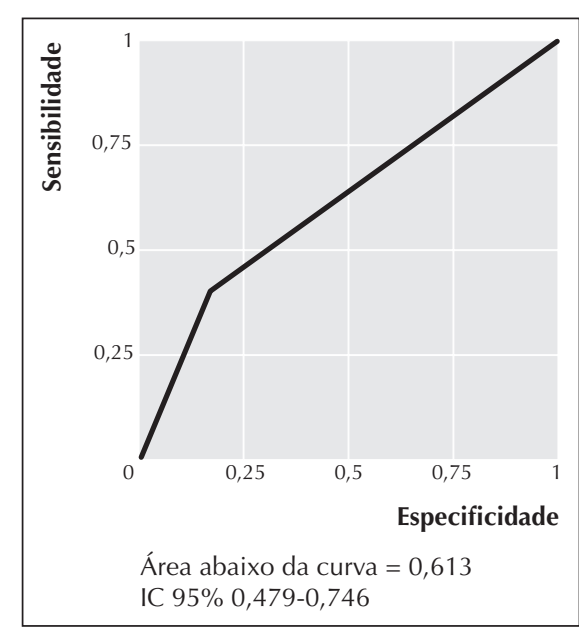

Figura 2. Curva ROC para ACL IgG.

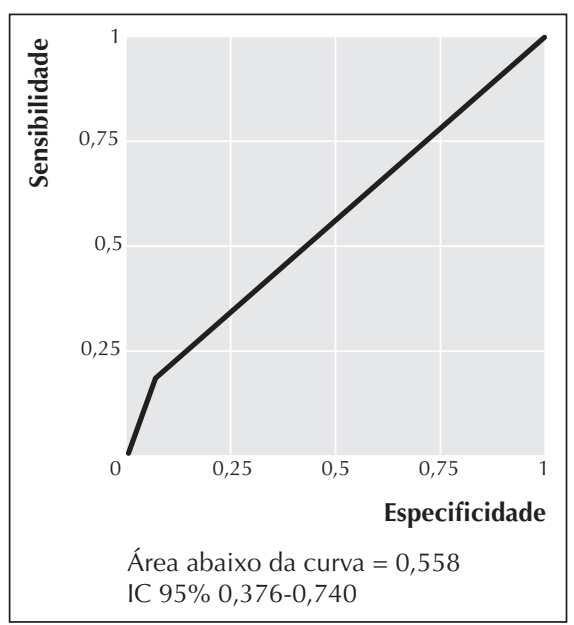

Figura 3. Curva ROC para LAC. merciais para a detecção de ACL de diferentes procedências. Também para a pesquisa de LAC, observa-se heterogeneidade de resultados em diferentes laboratórios e técnicas utilizadas. ${ }^{11-14}$ Consensos Internacionais para padronizar a pesquisa de anticorpos antifosfolipídios têm sido realizados para se melhorar a concordância interlaboratorial.A detecção de ACL através de um kit comercial no presente estudo demonstrou uma alta prevalência de ACL IgM em baixos títulos em indivíduos sem o diagnóstico de SAF, sugerindo sua alta sensibilidade para ACL IgM. Porém, a literatura demonstra que há uma tendência à associação entre resultados falso positivos e títulos baixos de ACL IgM, principalmente em indivíduos com fator reumatoide ou crioglobulinas. ${ }^{18-19}$

A descoberta de alta especificidade e maior área abaixo da curva ROC para o diagnóstico de SAF com a positividade do LAC em nosso estudo está de acordo com dados previamente relatados. ${ }^{3}$ A presença de LAC é o principal fator de risco para morbidade gestacional na SAF e para eventos trombóticos, independentemente do leito vascular ou do tipo de trombose, da presença de LES e do método utilizado para detectá-lo. ${ }^{3}$ Neste estudo, o encontro da positividade de LAC (12\%) foi inferior à observada na literatura (50-55\%). ${ }^{1,23-25}$ Este achado pode estar subestimado visto que sua pesquisa foi realizada em apenas 44 dos 106 participantes. Talvez o menor conhecimento sobre a importância do LAC como fator de risco para trombose e sua importância para o diagnóstico de SAF tenham contribuído para sua menor solicitação.

Em nosso estudo, a presença de ACL da classe IgG também apresentou alta especificidade para o diagnóstico de $\mathrm{SAF}$, o que está de acordo com a literatura. ${ }^{3}$ A presença de títulos moderados a altos de ACL da classe IgG é um fator de risco para trombose, mas com menor intensidade em relação ao LAC. ${ }^{3} \mathrm{O}$ grande número de indivíduos sem SAF e com ACL em baixos títulos observado no presente estudo se deve provavelmente ao baixo ponto de corte para positividade da ACL (10 unidades GPL, MPL ou APL) orientado pelo fabricante do kit. Como seria esperado, a maioria dos indivíduos sem SAF apresentou ACL IgM em baixos títulos. Entretanto, a diferença entre os grupos não foi significativa quanto à média de unidades MPL, pois alguns poucos indivíduos sem SAF apresentaram títulos elevados de ACL da classe IgM. Nos critérios de classificação de Sapporo, o ponto de corte utilizado para definir a positividade da ACL foi de 20 unidades GPL ou MPL. ${ }^{4}$ Recentemente, com o objetivo de se obter maior especificidade no diagnóstico de SAF, os critérios de Sapporo foram atualizados e o ponto de corte foi elevado para 40 unidades GPL ou MPL, ou níveis acima do percentil 99. ${ }^{5}$ A alta prevalência de anticorpos antifosfolipídios em indivíduos sem SAF também se deve ao fato de esses exames terem sido requisitados para pacientes com alguma suspeita clínica. A prevalência de anticorpos antifosfolipídios varia de $1 \%$ a 5,6\% na população geral sem quadro clínico suspeito do SAF e tende a aumentar com o passar da idade, principalmente em indivíduos com comorbidades. ${ }^{20}$

ACL da classe IgA só foi detectada em quatro indivíduos e, entre eles, apenas um paciente com SAF. Devido à baixa prevalência desse autoanticorpo, não foi possível analisá-lo separadamente. Esse isótipo não parece aumentar a sensibilidade para o diagnóstico de $\mathrm{SAF}, 5$ apenas ajuda a identificar subgrupos de pacientes, sendo altamente prevalente em pacientes afro-americanos com LES e se correlacionando com plaquetopenia, úlceras cutâneas e vasculite. ${ }^{21,22}$ 
Os pacientes com diagnóstico de SAF avaliados nesse estudo apresentam dados semelhantes aos da maioria dos estudos na literatura em relação à frequência do sexo feminino e à prevalência de trombose venosa, arterial e de morbidade obstétrica. ${ }^{1,23-25}$

Quanto aos anticorpos antifosfolipídios em pacientes com o diagnóstico de SAF, a prevalência de ACL da classe IgM foi de $92 \%$, superior aos $76,4 \%$ a $77 \%$ descritos em outros estudos $^{23,24}$ e muito acima dos $39,4 \%$ descritos em estudo realizado pelo mesmo autor utilizando a técnica de ELISA inhouse, ${ }^{25}$ o que confirma a alta sensibilidade da ACL IgM do kit analisado. Apesar de a ACL IgG ter sido mais específica para o diagnóstico de SAF neste estudo, sua prevalência de $40 \%$ foi inferior ao previamente descrito em pacientes com SAF $(75,7 \%$ a $81,6 \%){ }^{23-25}$

Em suma, nossos dados revelam a importância da positividade de LAC e de ACL da classe IgG no diagnóstico de SAF em indivíduos atendidos num hospital geral. Além disso, o presente estudo mostra que os anticorpos ACL do isótipo $\operatorname{IgM}$, a despeito de sua alta prevalência, bem como os do isótipo $\operatorname{IgA}$, não parecem contribuir para o auxílio diagnóstico da síndrome. 


\section{REFERÊNCIAS}

\section{REFERENCES}

1. Levine JS, Ware Branch D, Rauch J.The antiphospholipid syndrome. N Engl J Med 2002;346(10):752-763.

2. Roubey RAS. Immunology of the antiphospholipid syndrome. Arthritis Rheum 1996;39(9):1444-1454.

3. Galli M, Luciani D, Bertolini G, Barbui T. Lupus anticoagulants are stronger risk factors for thrombosis than anticardiolipin antibodies in the antiphospholipid syndrome: a systematic review of the literature. Blood 2003;101(5):1827-1832.

4. Wilson AW, Gharavi AE, Koike T, Lockshin MD, Branch $\mathrm{DW}$, Piette JC et al. International consensus statement on preliminary classification criteria for definite antiphospholipid syndrome. Report on an international workshop. Arthritis Rheum 1999;42(7):1309-1311.

5. Miyakis S, Lockshin MD, Atsumi T, Branch DW, Brey RL, Cervera $\mathrm{R}$ et al. International consensus statement on an update of the classification criteria for definite antiphospholipid syndrome. J Thromb Haemost 2005;4(2):295-306.

6. Brandt JT, Triplett DA, Alving B, Scharrer A. Criteria for the diagnosis of lupus anticoagulants: an update. On behalf of the Subcommittee on Lupus Anticoagulant/Antiphospholipid antibody of the Scientific Standardization Committee of the ISTH. Thromb Haemost 1995;74(4):1185-1190.

7. Pengo V, Biasiolo A, Bison E, Chantarangkul V, Tripodi A. Antiphospholipid antibody ELISAs: survey on the performance of clinical laboratories assessed by using lyophilized affinity-purified IgG with anticardiolipin and anti-beta2-Glycoprotein I activity. Thromb Res 2007;120(1):127-133.

8. Reber G, Arvieux J, Comby E, Degenne D, de Moerloose P, Sanmarco M et al. Multicenter evaluation of nine commercial kits for the quantitation of anticardiolipin antibodies. Thromb Haemost 1995;73(3):444-452.

9. Tincani A, Allegri F, Sanmarco M, Cinquini M, Taglietti M, Balestrieri $\mathrm{G}$ et al. Anticardiolipin antibody assay: a methodological analysis for a better consensus in routine determinations. A cooperative project of the European Antiphospholipid Forum. Thromb Haemost 2001;86(2):575-583.

10. Harris EN, Pierangelli S. Revisiting the anticardiolipin test and its standartization. Lupus 2002;11(5):269-275.

11. Exner T, Triplett DA, Taberner DA, Howard MA, Harris EN. Comparison of test methods for the lupus anticoagulant: international survey on lupus anticoagulants-I (ISLA-1). Thromb Haemost 1990;64(3):478-484.

12. Hemostasis Committee of the "Societé Française de Biologie Clinique": Laboratory heterogeneity of the lupus anticoagulant: a multicentre study using different clotting assays on a panel of 78 samples. Thromb Res 1992;66(4):349-364.

13. Goudemand J, Caron C, De Prost D, Derlon A, Borg JY, Sampol J et al. Evaluation of sensitivity and specificity of a standardized procedure using different reagents for the detection of lupus anticoagulants. The Working Group on Hemostasis of the Societé Française de Biologie Clinique and for the Groupe d'Études sur I'Hemostase et la Thrombose. Thromb Haemost 1997;77(2):336-342.

14. Jennings I, Kitchen S, Woods TA, Preston FE, Greaves M. Potentially clinically important inaccuracies in testing for the lupus anticoagulant: an analysis of results from three surveys of the UK National External Quality Assessment Scheme (NEQAS) for Blood Coagulation. Thromb Haemost 1997;77(5):934-937.

15. Tincani A, Allegri F, Balestrieri G, Reber G, Sanmarco M, Meroni P et al. Minimal requirements for antiphospholipid antibodies ELISAs proposed by the European Forum on antiphospholipid antibodies. Thromb Res 2004;114(5-6):553-558.

16. Pierangeli SS, Stewart M, Silva LK, Harris EN. Report of an anticardiolipin wet workshop during the VIIth International Symposium on antiphospholipid antibodies. J Rheumatol 1998;25(1):156-162.

17. Wong RCW on behalf of the Australasian aCL working party. Consensus guidelines for anticardiolipin antibody testing. Thromb Res 2004;114(5-6):559-571.

18. Bahar AM, Kwak JY, Beer AE, Kim JH, Nelson LA, Beaman KD et al. Antibodies to phospholipids and nuclear antigens in non-pregnant women with unexplained spontaneous recurrent abortions. J Reprod Immunol 1993;24(3):213-222.

19. Spadaro A, Riccieri V, Terracina S, Rinaldi T, Taccari E, Zoppini A. Class specific rheumatoid factors and antiphospholipid syndrome in systemic lupus erythematosus. Lupus 2000;9(1):56-60.

20. Petri M. Epidemiology of the antiphospholipid antibody syndrome. J Autoimmun 2000;15(2):145-151.

21. Tajima C, Suzuki Y, Mizushima Y, Ichikawa Y. Clinical significance of immunoglobulin A antiphospholipid antibodies: possible association with skin manifestations and small vessel vasculitis. J Rheumatol 1998;25(9):1730-1736.

22. Cucurull E, Gharavi AE, Diri E, Mendez E, Kapoor D, Espinoza LR. IgA anticardiolipin and anti-beta2-glycoprotein I are the most prevalent isotypes in African American patients with systemic lupus erythematosus. Am J Med Sci 1999;318(1):55-60.

23. Cervera R, Piette JC, Font J, Khamashta MA, Shoenfeld Y, Camps MT et al. For the Euro-Phospholipid Project Group: Antiphospholipid syndrome: clinical and immunologic manifestations and patterns of disease expression in a cohort of 1,000 patients. Arthritis Rheum 2002;46(4):1019-1027.

24. Mejia-Romero R, Garcia-Carrasco M, Galarza-Maldonado C, Santos P, Mendonza-Pinto C, Escárcega RO et al. Primary antiphospholipid syndrome in Latin American mestizo patients: clinical and immunologic characteristics and comparison with European patients. Clin Rheumatol 2008;27(7):891-897.

25. de Souza AWS, Silva NP, de Carvalho JF, D'Almeida V, Noguti MAE, Sato EI. Impact of hypertension and hyperhomocysteinemia on arterial thrombosis in primary antiphospholipid syndrome. Lupus 2007;16(10):782-787. 